\title{
Effects of a Whole-body Vibration on Fatigue-pain and Mobility Among Adults and Middle-aged People
}

\section{Fayz S Alshahry ${ }^{1 *}$, Alya A Alalwan ${ }^{2}$, Latifah M Alruwaitea ${ }^{2}$, Latifa M Alshawi $^{2}$ and Lamia A Alhussain ${ }^{2}$}

${ }^{1}$ Assistant Professor, Consultant Rehabilitation, Saudi Arabia

${ }^{2} J u n i o r$ Occupational Therapists, Saudi Arabia

*Corresponding Author: Fayz S Alshahry, Assistant Professor, Consultant

Rehabilitation, Saudi Arabia.
Received: May 30, 2021

Published: June 25, 2021

(C) All rights are reserved by Fayz S Alshahry., et al.

\begin{abstract}
Introduction: Whole body vibration (WBV) is a device that conducts oscillatory motion. WBV stimulates muscle spindles' primary motor endings in muscles which rises primary afferent neurons sensory input led to produce powerful alpha motor neuron output, result in increased recruitment rate of motor units that improve muscles activity. WBV does not cause any harmful side effect unless exposed to it with high frequency for a long period of time.

Aim: The aim of this study was to detect the effects of WBV on decreasing Fatigue-Pain (FP) and increasing mobility after exercise among healthy individuals.

Methodology: This is quasi-experimental study that have been carried at KSAU-HS Sport Complex. We examined participants' pain and mobility before and after WBV. fatigue was measured using visual analogue scale, while mobility was measured by fingertip-tofloor test. The target participants are individuals with low threshold fatigue, aged between 18-59 males and females. Participants stood on the device in semi-squad position for 5 minutes, and the frequency increased gradually from 10-50 $\mathrm{Hz}$.

Results: one hundred ninety-two healthy individuals with average age 21 . By using chi-square, females represented only $29.5 \%$ of total participants, while males represented 70.5\%. The results showed that the average pain before WBV was 4.0 , which has decreased to 2. after WBV. Moreover, the mobility median before WBV was ( $-0.5 \mathrm{inch})$, which has increased to ( $-1.20 \mathrm{inch})$. Overall results prove the effect of WBV on mobility and Fatigue pain. The effect was tested using statistical test such as chi-square and Wilcoxon Signed Rank Test. A p value less than 0.05 was considered as significant. However, the study showed variation on individual basis, but did not reach a significant level.

Conclusion: Results has proved significant level of decreasing FP and increasing mobility after WBV. The specification of vibration effect still debatable, the study suggests integrated effect of vibratory analgesia and lateral inhibition which directly could affect on the FP and mobility temporarily, and respectively.
\end{abstract}

Keywords: Vibration; Fatigue Pain (F. P); Mobility; Middle-aged; Adult Healthy

\section{Introduction}

Some adults and middle-aged people (18 - 59 years old) suffer fatigue and pain in muscles after exercising [1,2]. Reducing pain and increasing mobility after exercises can be managed by using whole body vibration (WBV) for maintaining and improving quality of life and health among adults and middle-aged people [1,3]. 
WBV is a device that conducts oscillatory motion which generates different types of effects [4]. It stimulates muscle spindles' primary motor endings in muscles which rises primary afferent neurons sensory input led to produce powerful alpha motor neuron output, result in increased recruitment rate of motor units that improve muscles activity $[4,6]$. Previous studies have shown that WBV has a significant effect on relieving pain. Also, other studies have proved that WBV has a significant effect on increasing mobility $[2,4,7,8]$. In contrast, some studies demonstrated that exposing to high frequency of WBV for long period of time can lead to health problems such as gastrointestinal problems, visual and vestibular disturbances, low back pain, prostate disorders, and peripheral nervous system dysfunction $[9,10]$.

WBV has a fundamental method based on the natural gait pattern of human beings. As it stimulates movement similar to human gait pattern by promoting side-alternating motion like a seesaw movement with different amplitude and frequency. The main principle is to imitate the human gait pattern by producing rapid movement of the platform that encourages movement like walking, causing a high frequency tilting pelvis movement in response to rhythmic muscle contraction. The platform transfers energy to the human body via sinusoidal vibrations intervals [4]. Frequency starts at 10 hertz onward causing muscle contraction with no conscious. As a reflection, it activates leg muscles, stomach muscles, and the back muscles. The adjustable training frequency determines the number of stretches that reflex the contractions per second. The frequency in Hertz (cycles per second) is set on the device and can be changeable according to the training objectives. To illustrate, low frequencies are used for mobilization, intermediate frequencies are used to train muscle function, and high frequencies are used to increase muscle performance. Furthermore, the amplitude is the change in the deflection platform upwards or downwards, and it depends on the foot position.

There are two types of WBV effects which can be divided according to time of effect existing. These types are short-term (immediate) and long-term. Short-term effects are the effects that exist immediately after undergoing WBV. The immediate effect of WBV activates more motor units and muscle fibers in conscious muscle contractions than in normal muscle. As result, muscles will be stimulated more efficiently and will be able to produce extra force [11]. In this process, to minimize fatigue and increase performance, the effect of stimulus must not be prolonged and the intense of it should be low. The second immediate effect of WBV enhances blood circulation due to rapid muscles' contraction and relaxation at 20 to 50 times per second [12]. As result of vasodilation after applying WBV, clients will undergo warm sensation, tingling, and prickling at skin level [13].

The long-term principle is applying vibration after the body experiences fatigue or stress. As the body is toughly overloaded the super compensation principle is applied, which causes the body to adapt to loading, thus increases performance. Some studies have concluded that WBV training can increase strength which can be achieved in the area of explosive power [14]. There is no overloading and no additional weight in WBV which ensure that there is little loading to bones, ligaments, and joints. Therefore, WBV is ideal for elderly and people with illnesses or disorders who has difficulty in training, and it is suitable for athletes for muscle stimulating and strengthening without overloading the physical system $[15,16]$. Besides its effect on the muscles, WBV also has a positive influence on mineral density of bone. Vibrations cause compression and remodeling of the bone tissue, activating the osteoblasts, while decreasing the activity of osteoclasts [17]. Bone mineral density will increase over time due to repeated stimulation of the system combined with the increased pull on the bones by the muscles. As the nutrients supply gets better, it enhances the circulation, related bone perfusion, and penetrate the bone tissue, these are contributing factors [18]. In Berlin Bedrest Study, it was mentioned that exposing to vibration for 10 minutes for six times per week prevents muscle and bone loss which is a result of bedrest more than 55 days [19].

Due to lack studies that evaluate the effects of whole-body vibration among healthy individuals within the community, the aim of this study was to detect the effects of whole-body vibration on pain and mobility among adults and middle-aged people after exercising. It was assumed that undergoing WBV would decrease the F.P and increase the mobility in the adult and middle-aged people. Benefits of this study were to develop the knowledge and to recommend WBV to be offered in sport centers after exercising instead of stretching exercise.

\section{Methodology}

This study was conducted at Sport Complex at King Saud bin Abdulaziz University for Health Sciences (KSAU-HS) in Kingdom of Saudi Arabia (Riyadh). Sport Complex was established in 2015 for university staff and students. This sport center provides different kind of sports such as football, basketball, and swimming. Also, it 
has different facilities such as basketball court, football yard, tennis court, and swimming pool. We chose this sport center because of age rang that meets the research's need.

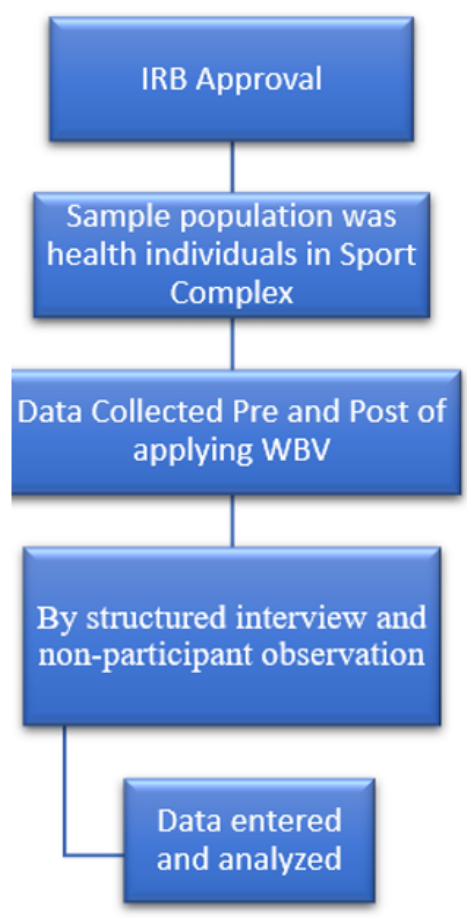

Figure a

The sample size was collected from sport complex at KSAU-HS. Based on recorded from the sport center the population registered is around 940 individuals who are mainly 880 males either students or staff registered in their facility. The minimum required sample size was 120 healthy individuals according to $7 \%$ margin of error and $90 \%$ confidence level, calculated by Raosoft online sample size calculator.

This study was conducted by using convenience sampling which is a type of non- probability sampling. It depended on participates' and researchers' convenience. Subjects collected after inviting them, explaining to them the procedure of study, and taking their consent.

First, we asked the participants who regularly attend the Sport Complex for exercising to participate voluntarily. If participants accept, they sign the consent form. Then, the researchers followed the following steps.

After participant finished from his/her regular exercising, their FP and mobility were obtained. The FP was collected by structured interview which was Visual Analogue Scale (VAS), and mobility was collected by non-participant observation which was done by fingertip-to-floor test.
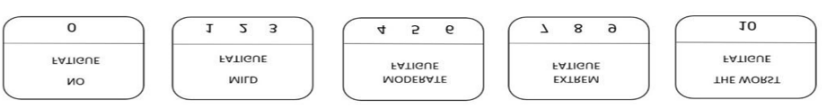

Figure b
Participants were included if they fulfilled the following criteria: normal healthy people and aged between 18 and 59 years old. As the majority of subjects who attend the sport centers are between 18 - 40 years old. Individuals were excluded if they have been diagnosed with vertebral column problems such as scoliosis neuromuscular diseases, heart diseases such as heart arrhythmia, rheumatoid arthritis, osteoarthritis, recent fracture, were taken medication known to affect muscle strength such as anabolic steroids, were fitted with cardiac pacemaker, or had already received WBV treatment. Females in period or pregnancy time were temporary excluded until these conditions disappear.

This study is considered quasi-experimental approach (pretestposttest design) which helped to estimate the causal impact of WBV with fatigue-pain and mobility on healthy individuals. Subjects' fatigue-pain and mobility were measured repeatedly both before and after applying the vibration measurement.
After that, participants were asked to stand on WBV device in semi-squad position for five minutes. The frequency started at 10 $\mathrm{Hz}$ and gradually increased with each minute until it reached 50 $\mathrm{Hz}$ in the fifth minute. The semi-squad position (Figure 1) is the optimal position that makes the lower extremities in stretching position, so WBV can work effectively on each muscle.

Participants who felt numbness after WBV, thus we requested participants to have two minutes break until the numbness disappeared. Then, the pain and mobility were obtained again by using the same measurements tool.

The independent variable of this study is exposure to WBV, and dependent variables are FP and mobility. There were two measurement tools for the investigation of fatigue-pain and mobility. 
Fatigue-Pain was measured by VAS which is valid and reliable (1). Participants were asked to determine the FP degree from (0) to (10), where (0) means no fatigue, (5) moderate, and (10) severe fatigue. Mobility was assessed by valid and reliable test which was fingertip-to-floor test (2). In fingertip-to-floor test, subject was asked to stand erect with feet together and shoes removed, then subject was asked to bend forward with maintaining his/her arms, fingers, and knees are extended as much as he/she can for three times. The distance from middle fingertip to floor was measured by using tape measure in inch. If subject can reach the floor and more, we measure the mobility by asking him/her to place palm of hand in floor as much as he/she can and measure what his/her palm distance that touch the floor. In fingertip-to-floor test, the more decrease in number, the more mobility the participant has, then he differences in the two measurement will be considered.

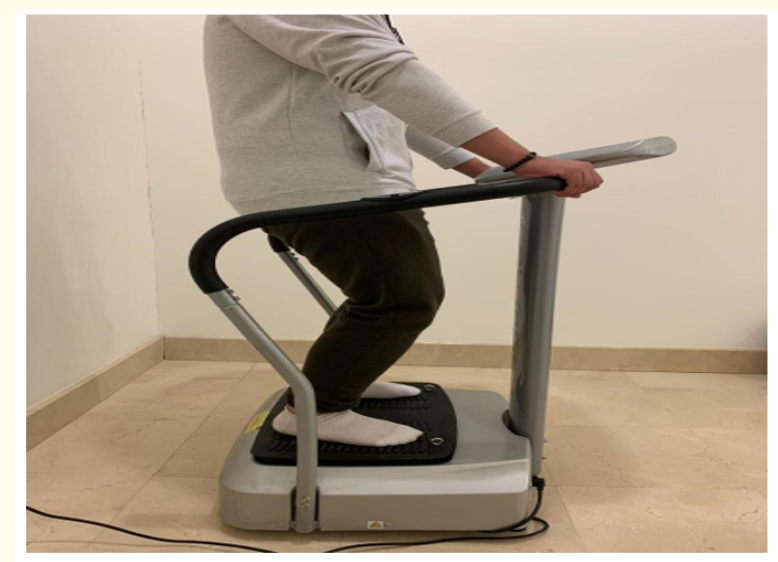

Figure 1: The semi-squad position on the vibrator platform.

The collected data was entered by using excel sheet and analyzed by using SPSS. The results were representing by using tables and figures. The continues variables were normally distributed, so the variables were described by using mean standard deviation. Also, for categorical variables, frequency and percentage were used to represent it. Various statistical test such as chi-square and Wilcoxon Signed Rank Test were employed to analyze the data. A p value less than 0.05 was considered as significant.

\section{Results}

The study sample size of this study is 120 , however the number of participants were 129 . As the table 1 shows, the median of the age was 21 . The maximum age of participants was 56 years, while the minimum age was 18 years. Table 2 shows that females represented only $29.5 \%$ of total participants, while males represented $70.5 \%$. The median F. pain level before WBV was 4.00, with Interquartile range (IQR) was 4.00, which decreased to 2.00 after WBV with IQR of 3.00 as shown in table 3. The median for the mobility before WBV was -0.50 inch with IQR of 5.83, which increased to -1.20 inches with IQR of 4.63 as shown in table 3 . The result showed a significant effect on increasing mobility and decreasing $\mathrm{F}$. pain among participants with $\mathrm{P}$ value of 0.001 for both fatigue and mobility as shown in table 3 .

As shown on table 3, Effect of Whole-Body Vibrator (WBV) with test statistic and $p$ value. FP Median and Inter Quartile Range, Mobility Median and range were calculated. Table 4 showed the influence of gender Influence of Gender to post exposure to F. pain (n =129).

\begin{tabular}{|l|c|}
\hline & Age \\
\hline Minimum & 18 \\
\hline Maximum & 56 \\
\hline Median & 21 \\
\hline IQR (Interquartile range) & 4 \\
\hline
\end{tabular}

Table 1: Age distribution.

\begin{tabular}{|l|c|c|}
\hline & Male & Female \\
\hline Frequency & 91 & 38 \\
\hline Percentage & $70.5 \%$ & $29.5 \%$ \\
\hline
\end{tabular}

Table 2: Gender frequency.

\begin{tabular}{|c|c|c|c|c|c|}
\hline $\begin{array}{c}\text { Para } \\
\text { meter }\end{array}$ & $\begin{array}{l}\text { Statistics } \\
\text { used }\end{array}$ & $\begin{array}{c}\text { Pre } \\
\text { exposure to } \\
\text { WBV }\end{array}$ & \begin{tabular}{|c|} 
Post \\
exposure \\
to WBV
\end{tabular} & $\begin{array}{c}\text { Test } \\
\text { Statistic }\end{array}$ & p value \\
\hline \multirow[b]{2}{*}{ FP } & Median & 4.0 & 2.0 & \multirow[b]{2}{*}{$Z=-6.131$} & \multirow[b]{2}{*}{$0.001^{*}$} \\
\hline & $\begin{array}{c}\text { Inter Quartile } \\
\text { Range }\end{array}$ & 4 & 3 & & \\
\hline \multirow{2}{*}{ Mobility } & Median & -0.5 inch & -1.2 inch & \multirow{2}{*}{$Z=-8.038$} & \multirow{2}{*}{$0.001^{*}$} \\
\hline & Range & 5.83 inch & 4.63 inch & & \\
\hline
\end{tabular}

Table 3: Effect of whole-body vibrator (WBV) with test statistic and $\mathrm{p}$ value.

Test used is Wilcoxon Signed Rank Test. $I n c h=2.54 \mathrm{~cm}$. mobility measure is the difference between pre-vibration and post vibration.

*Significant at 0.05 . 


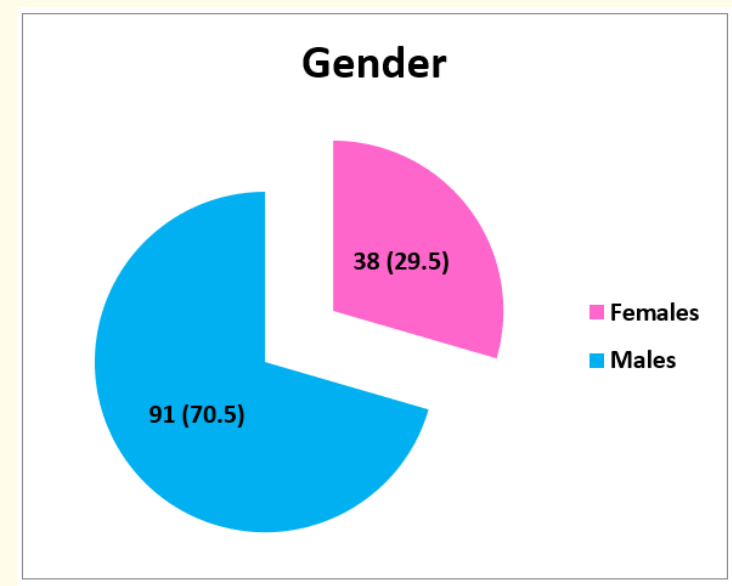

Figure 2: Gender distribution, fast majority are for male.

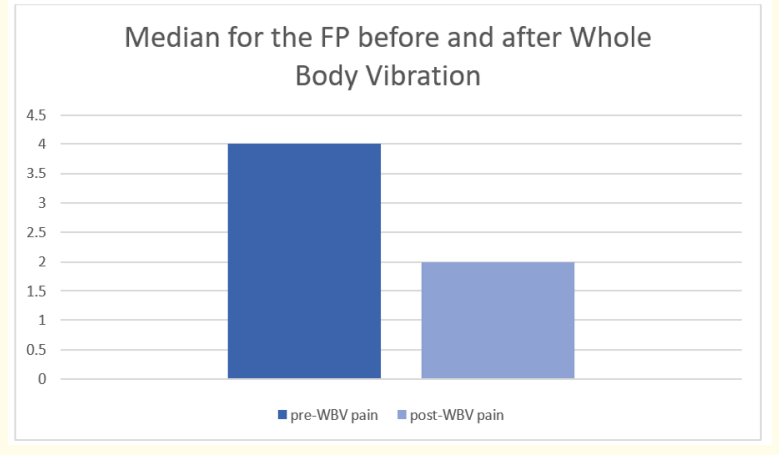

Figure 3: FP before and after. Test Statistic $(\mathrm{Z}=-6.131)$, and P-value (0.001).

Test used is Wilcoxon Signed Rank Test.

*Significant at $\mathrm{P}<0.05$.

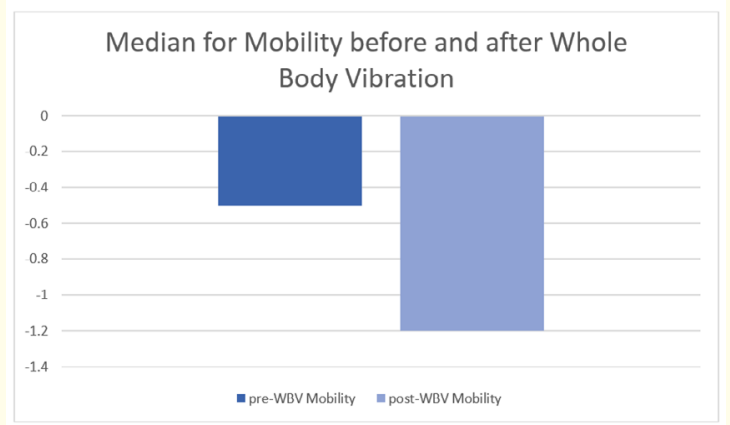

Figure 4: Mobility pre and post vibration. Test Statistic ( $\mathrm{Z}=$-8.038), and P-value (0.001*).

Test used is Wilcoxon Signed Rank Test.

*Significant at $\mathrm{P}<0.05$.

\begin{tabular}{|c|c|c|c|c|c|}
\hline \multirow{2}{*}{$\begin{array}{l}\text { Pain and } \\
\text { mobility }\end{array}$} & Variable & \multirow{2}{*}{$\begin{array}{c}\text { Sample } \\
\text { size }\end{array}$} & \multirow{2}{*}{$\begin{array}{c}\text { Mean } \\
\text { rank }\end{array}$} & \multirow{2}{*}{$\begin{array}{c}\text { Test } \\
\text { statistic }\end{array}$} & \multirow{2}{*}{$P$ value } \\
\hline & Gender & & & & \\
\hline \multirow[b]{2}{*}{ Pre-F. pain } & Male & 91 & 68.32 & \multirow[b]{2}{*}{$\mathrm{U}=1426.5$} & \multirow[b]{2}{*}{0.115} \\
\hline & Female & 38 & 57.04 & & \\
\hline \multirow[b]{2}{*}{ Post F. pain } & Male & 91 & 66.98 & \multirow[b]{2}{*}{$\mathrm{U}=1549.0$} & \multirow[b]{2}{*}{0.346} \\
\hline & & 38 & 60.26 & & \\
\hline \multirow{2}{*}{$\begin{array}{l}\text { Pre } \\
\text { mobility }\end{array}$} & Male & 91 & 58.40 & \multirow{2}{*}{$U=1128.5$} & \multirow{2}{*}{$0.002 *$} \\
\hline & Female & 38 & 80.80 & & \\
\hline \multirow{2}{*}{$\begin{array}{l}\text { Post } \\
\text { mobility }\end{array}$} & Male & 91 & 58.39 & \multirow{2}{*}{$\mathrm{U}=1127.5$} & \multirow{2}{*}{$0.002^{*}$} \\
\hline & Female & 38 & 80.83 & & \\
\hline
\end{tabular}

Table 4: Influence of gender to post exposure to F. pain $(n=129)$.

*Significant at $5 \%$.

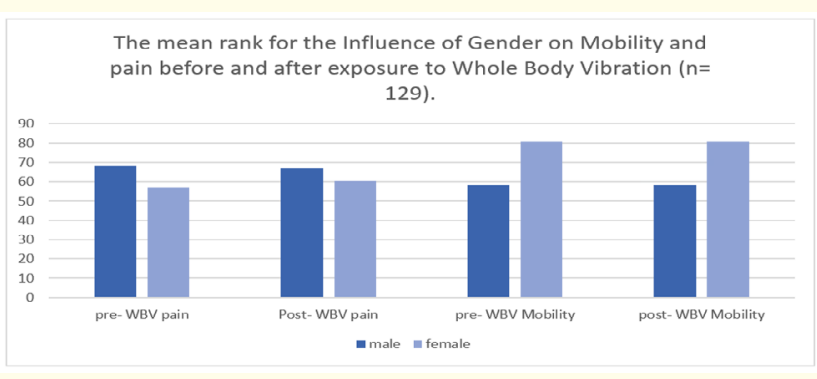

Figure 5: The mean rank of influence of gender on mobility and F. pain. indicate mobility range in female is much higher than in male.

Test Statistic for pre- WBV F. Pain $(\mathrm{U}=1426.5)$, and P-value (0.115).

Test Statistic for post- WBV F. Pain ( $\mathrm{U}=1549.0)$, and P-value (0.346).

Test Statistic for pre- WBV Mobility (U = 1128.5), and P-value $\left(0.002^{*}\right)$.

Test Statistic for post- WBV Mobility (U = 1127.5), and P-value $\left(0.002^{*}\right)$.

*Significant at $\mathrm{P}<0.05$.

\section{Discussion}

This study investigated the effectiveness of whole-body vibration in increasing mobility and decreasing fatigue pain (FP) after exercising on healthy individuals. To our knowledge, this is the first study that evaluates the effect of WBV among healthy individuals within the community. We conducted the study on one 
hundred twenty-nine males and females between eighteen and fifty-nine years with median age of 21y. The effect was tested using chi-square and Wilcoxon Signed Rank Test that indicated a significant increase in mobility and decrease in pain. The overall results proved the effect of WBV in increasing mobility and decreasing pain. However, the study showed variation in pain and mobility on individual basis, overall result significance was shown in table 3 , and 4 .

The participants showed variation upon F. pain and mobility. In F. pain, there were 15 participants showed no differences in pain pre and post WBV, and there were 14 participants had their pain increased after WBV. In mobility, there were 14 participants showed no differences in mobility pre and post WBV, and there were 4 participants had their mobility decreased after WBV. However, there were around 6 participants decided not to continue the study due to getting tired, some were in rush, or due to pain. Female clients showed a bigger rang of mobility and less susceptibility to FP changes compared to males.

Generally, adult and middle-aged people suffer fatigue and pain in muscles after exercising due to sarcopenia. Sarcopenia is a decrease in muscle mass and strength due to normal aging process, which leads to decline mobility and increase pain in muscles [1,2]. WBV is applied after exercise to improve mobility which will decrease pain and fatigue. Previous studies have shown that WBV has a significant effect on releasing pain and increasing mobility $[2,4,7,8]$.

The fatigue-pain was measured before and after WBV using Visual Analogue Scale (VAS). Majority of the participants' fatigue-pain was significantly decreased after using whole body vibration. Previously published studies have proven that applying WBV after exercises will stimulate muscles efficiently, thus muscles will be able to produce extra force [11]. Other studies suggested that to minimize fatigue and increase performance, the effect of stimulus must not be prolonged and the intense of it should be low [12]. In this study, the median fatigue-pain before whole body vibration was 4 , which decreased to average fatigue-pain after whole body vibration to 2. However, several participants have their pain increased after WBV, and others showed no differences in pain pre and post WBV. For those who showed increased pain were reported centralized pain in certain parts. This might be due to neuor-irretation or due to overstimulation of minor neglected traumas.

The other variable is mobility which was measured by fingertipto-floor test before and after using whole body vibration. Test is positive if subject cannot reach floor and negative if subject can reach floor and further. Many participants' mobility was significantly increased after whole body vibration. According to previous studies, a significant reduction in soreness and increase in flexibility after applying WBV post-exercised $[22,23]$. Furthermore, when using WBV as a recovery aid, it showed improvement in flexibility $[24,25]$. In this study, the median mobility increased from -0.5 inch to -1.2 inch after applying the whole-body vibration. In contrast, some participant showed no difference in mobility pre and post WBV, while others showed less or decreased mobility after WBV. As participants were asked to reach the floor with palm of hand, some participants showed no difference in their mobility since they already can place their palm fully in floor.

The temporary effect of vibration in mobility could be due to the vibratory analgesia effect [26]. The explanation for this phenomenon is most likely to be that it is mediated by lateral inhibition at the segmental level on spinal cord, in pain-coding cells with centersurround receptive zones.

There is a little evidence too that support the view that distraction is the primary mechanism of vibratory analgesia. The results are more consistent with a recently proposed theory of interactions between two cortical areas that are primarily involved in coding pain [26]. The study believes on integrated effect of vibratory analgesia and lateral inhibition which directly could affect on the FP and mobility temporarily, respectively.

\section{Strength}

In this study, WBV showed effectiveness in positive manners in reducing fatigue-pain and increasing mobility among participants. The participants represented healthy individuals in Sport complex center; therefore, we can generalize our findings to be used in the sport activities.

\section{Limitations}

The current study was conducted in one place which was the Sport complex center for a short period of time; therefore, we were restricted to limited community who mainly were students and staff of King Saud bin Abdulaziz University for Health Sciences with limited wide range of ages. When comparing between participants, there were shortage in female participants due to sport complex center schedule that allows two days for females. Moreover, females have more precautions than males like pregnancy and period which made it difficult in conducting large sample of female. On the other hand, fingertip-to-floor test was measured without 
applying a step for participants to stand over it for an accurate mobility measurement.

\section{Conclusion and Recommendation}

A post-exercise WBV showed a sufficient effect on reducing selfrating fatigue-pain compared to pre WBV pain. Also, there was a remarkable increase in mobility among participants after applying WBV. The condition of applying WBV post-exercise resulted in overall increasing mobility and decreasing fatigue. Furthermore, a regular post-workout program of WBV may enhance the recovery after exercise with taking into consideration different exercises as well as WBV frequencies and duration. For future studies, we suggest that having wide range of ages and large community.

\section{Bibliography}

1. Goudarzian M., et al. "Mobility, balance, and muscle strength adaptations to short-term whole body vibration training plus oral creatine supplementation in elderly women". Asian Journal of Sports Medicine 8.1 (2017): 1-9.

2. Goudarzian M., et al. "Effects of whole body vibration training and mental training on mobility, neuromuscular performance, and muscle strength in older men". Journal of Exercise Rehabilitation 13.5 (2017): 573-580.

3. Siparsky PN., et al. "Muscle Changes in Aging: Understanding Sarcopenia". Sports Health 6.1 (2014): 36-40.

4. Merrigan J., et al. "Effect of Post-Exercise Whole Body Vibration with Stretching on Mood State, Fatigue, and Soreness in Collegiate Swimmers". Sports 5.1 (2017): 7.

5. Activity M and Instability CA. "Effects of Whole Body Vibration Exercise on Lower Extremity Muscle Activity and Balance Ability in Football Player with Chronic Ankle Instability". Journal of the Korean Physical Society 29.6 (2017): 293-298.

6. Lam FMH., et al. "Effects of adding whole-body vibration to routine day activity program on physical functioning in elderly with mild or moderate dementia: a randomized controlled trial". International Journal of Geriatric Psychiatry 33.1 (2018): 21-30.

7. Milanese C., et al. "Ten-week whole-body vibration training improves body composition and muscle strength in obese women". International Journal of Medical Sciences 10.3 (2013): 307-311.
8. Pérez-Turpin JA., et al. "Effects of whole body vibration on strength and jumping performance in volleyball and beach volleyball players". Biology of Sport 31.3 (2014): 239-245.

9. Yung M., et al. "The combined fatigue effects of sequential exposure to seated whole body vibration and physical, mental, or concurrent work demands". PLoS One 12.12 (2017): 1-20.

10. Zeeman ME., et al. "Whole-body vibration induces pain and lumbar spinal inflammation responses in the rat that vary with the vibration profile". Journal of Orthopaedic Research 34.8 (2016): 1439-1446.

11. Delecluse C., et al. "Effects of whole body vibration training on muscle strength and sprint performance in sprint-trained athletes". International Journal of Sports Medicine 26.8 (2005): 662-668.

12. Lohman EB., et al. "The effect of whole body vibration on lower extremity skin blood flow in normal subjects". Medical Science Monitor 13.2 (2007): CR71-76.

13. Stewart JM. "Plantar vibration improves leg fluid flow in perimenopausal women". AJP-Regulatory, Integrative and Comparative Physiology 288.3 (2004): R623-629.

14. Delecluse C., et al. "Strength increase after whole-body vibration compared with resistance training". Medicine and Science in Sports and Exercise 35.6 (2003): 1033-1041.

15. Cochrane DJ and Stannard SR. "Acute whole body vibration training increases vertical jump and flexibility performance in elite female field hockey players". British Journal of Sports Medicine 39.11 (2005): 860-865.

16. Mahieu NN., et al. "Improving strength and postural control in young skiers: Whole-body vibration versus equivalent resistance training". Journal of Athletic Training 41.3 (2006): 286293.

17. Frost HM. "Defining osteopenias and osteoporoses: Another view (with insights from a new paradigm)". Bone 20 (1997): 385-391.

18. Jordan J. “Good vibrations and strong bones?” American Journal of Physiology - Regulatory, Integrative and Comparative Physiology 288 (2005): 555-556. 
19. Bleeker MWP. "Vascular adaptation to deconditioning and the effect of an exercise countermeasure: results of the Berlin Bed Rest study". Journal of Applied Physiology 99.4 (2005): 12931300 .

20. Farrar JT., et al. "Validity, reliability, and clinical importance of change in a 0-10 numeric rating scale measure of spasticity: a post hoc analysis of a randomized, double-blind, placebo-controlled trial". Clinical Therapy 30.5 (2008): 974-985.

21. Perret C., et al. "Validity, reliability, and responsiveness of the fingertip-to-floor test". Archives of Physical Medicine and Rehabilitation 82.11 (2001): 1566-1570.

22. Herbert RD., et al. "Stretching to prevent or reduce muscle soreness after exercise". Cochrane Database of Systematic Reviews 7 (2011).

23. Perrier ET., et al. "The acute effects of a warm-up including static or dynamic stretching on countermovement jump height, reaction time, and flexibility". The Journal of Strength and Conditioning Research 25.7 (2011): 1925-1931.

24. Woods K., et al. "Warm-up and stretching in the prevention of muscular injury". Sports Medicine 37.12 (2007): 1089-1099.

25. Gerodimos V., et al. "Whole-body vibration training in middleaged females: improving muscle flexibility and the power of lower limbs". Sport Sciences for Health 11.3 (2015): 287-294.

26. Mark Hollins., et al. "How does vibration reduce pain?" Perception, SAGE Journals 43.1 (2014): 70-84.

Volume 4 Issue 7 July 2021

(C) All rights are reserved by Fayz S Alshahry., et al. 